EPJ Web of Conferences 19, 08003 (2012)

DOI: $10.1051 /$ epjconf/20121908003

(C) Owned by the authors, published by EDP Sciences, 2012

\title{
The Milky Way and its gas: Cold fountains and accretion
}

\author{
F.J. Lockman ${ }^{\mathrm{a}}$ \\ National Radio Astronomy Observatory, PO Box 2, Green Bank, WV 24944, USA
}

\begin{abstract}
The Milky Way is acquiring gas from infalling high-velocity clouds. The material enters a diskhalo interface that in many places is populated with HI clouds that have been ejected from the disk through processes linked to star formation. The Smith Cloud is an extraordinary example of a high-velocity cloud that is bringing $>10^{6} \mathrm{M}_{\odot}$ of relatively low metallicity gas into the Milky Way. It may be part of a larger stream, components of which are now passing through the disk.
\end{abstract}

\section{INTRODUCTION}

The disk-halo transition zone in spiral galaxies is a lively place. Energetic events associated with star formation can lift gas several kpc into the halo [9, 14]. Some spirals have an associated population of extra-planar HI clouds that are probably being accreted from elsewhere [4, 20]. Gas stripped from dwarf galaxies can add to the environment as well. These phenomena give many spirals a ragged outer HI envelope with a variety of kinematics beyond simple circular rotation [15]. Recent studies of the Milky Way have provided new insight into these processes that shape the evolution of galaxies.

\section{HI CLOUDS IN THE DISK-HALO INTERFACE}

The discovery of a population of discrete neutral hydrogen clouds extending outward from the disk several kpc into the halo raises a number of questions, among them whether the clouds are recent condensations from a hot galactic halo, or a component of the disk ISM ejected from the plane $[10,18,19]$. The "disk-halo" clouds are rotating with the Galactic disk, and their numbers decrease exponentially with distance from the plane, but their cloud-cloud velocity dispersion, if representative of an isotropic random motion, is nearly an order of magnitude too small to account for the clouds' scale height.

Recently, several hundred of these clouds were analyzed using data from a uniform survey of two regions symmetric about the line of sight to the Galactic Center - one in the first quadrant of longitude (QI) and another in the fourth (QIV) [2, 3]. The observations were compared with simulations that properly account for various biases including distance errors and confusion. The derived vertical distribution of the disk-halo HI clouds shown in Figure 1 reveals a major anomaly: while the cloud-cloud velocity dispersion (and indeed all properties of individual clouds) is identical in the two quadrants, both the number of clouds and their scale height differs significantly. After ruling out possible systematic effects, Ford et al. [3] conclude that the cloud numbers and scale-height are related to star formation: the location chosen for study in Quadrant I coincidentally includes an area with many HII regions, while the longitude-symmetric area in Quadrant IV lies between major spiral arms.

\footnotetext{
a The National Radio Astronomy Observatory is operated by Associated Universities, Inc., under a cooperative agreement with the National Science Foundation. e-mail: jlockman@nrao.edu

This is an Open Access article distributed under the terms of the Creative Commons Attribution-Noncommercial License 3.0, which permits unrestricted use, distribution, and reproduction in any noncommercial medium, provided the original work is properly cited.
} 


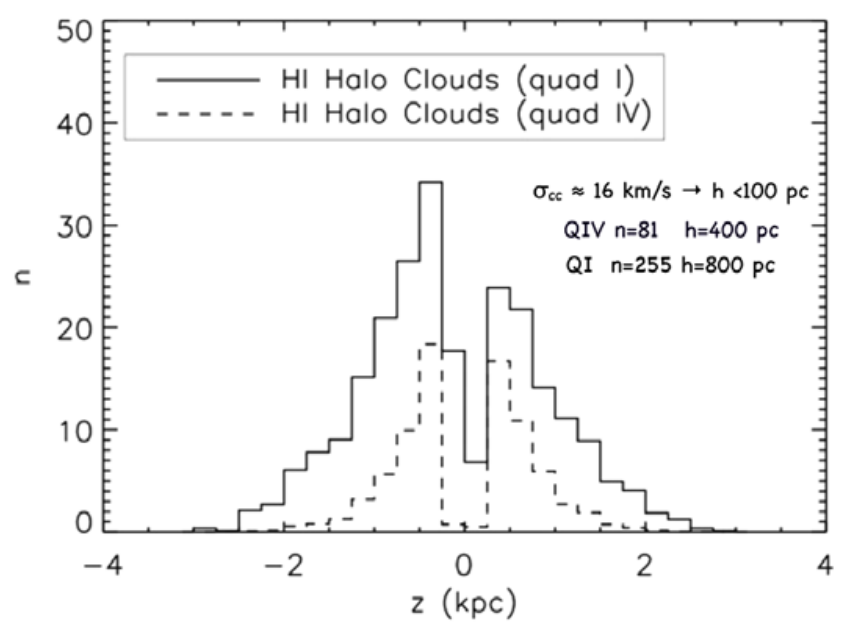

Figure 1. Observed number of disk-halo HI clouds as a function of distance from the Galactic plane for samples in identical volumes of the First and Fourth Galactic quadrants (adapted from Ford et al. [3]). QI has more clouds and a larger scale height than QIV, while the cloud-cloud velocity dispersion is identical and too small to account for even the smaller scale height. The decrease in cloud numbers near the plane results from confusion.

The disk-halo clouds may thus be created through the breakup of large superbubbles, indeed, some clouds are arranged in long chains that stretch out of the disk, perhaps analogs to the dust pillars of NGC 891 [8]. How these might evolve into discrete HI clouds is not known. In the classic picture of the galactic fountain, hot gas heated by supernovae rises buoyantly then cools, condenses and returns to the disk [7]. The disk-halo clouds appear more likely to have been lofted away from the plane in structures that exist for perhaps 10 Myr before breaking up [14]. They may be the products of a galactic fountain, but the flow is in cool neutral rather than hot ionized gas.

\section{GAS FLOWS INTO THE MILKY WAY}

It has long been suggested that the high-velocity HI clouds (HVCs) that cover much of the sky are accreting from a hot halo that is either a relic of the formation of the Milky Way or is powered by a galactic fountain. Some HVCs might also be the ISM stripped from satellite galaxies, although the Magellanic Stream is the only convincing local example. The low metallicity of many HVCs precludes their formation in a galactic fountain, but the origin and fate of most HVCs is still unknown [16, 21, 22]. Recent observations of the HVC known as the "Smith Cloud" [17] show that it is an exceptional object, one that might give general insight into the way that the Milky Way continues to acquire gas.

\subsection{The Smith Cloud}

Figure 2 shows an HI channel map of the Smith Cloud from a new survey made with the 100 meter Green Bank Telescope (GBT) of the NRAO at an angular resolution of 9', observed and reduced as described by [1]. Properties of the Cloud are summarized next to the Figure [5, 11]. This HVC is unique in that both its distance and its full space motion are known, and while it has only recently begun to be studied in detail, what we have learned already is very informative.

The Smith Cloud has a total velocity $\sim 300 \mathrm{~km} \mathrm{~s}^{-1}$, so it is gravitationally bound to the Milky Way. The largest velocity component is in the direction of Galactic rotation. It lies interior to the Solar Circle at $\mathrm{R}_{\text {gal }}=7.4 \mathrm{kpc}$, and is moving upwards toward the Galactic plane, which it will cross in about $30 \mathrm{Myr}$ at $\mathrm{R}_{\text {gal }} \approx 11 \mathrm{kpc}$ from the Galactic center. The tip of the cloud at $z=-2.2 \mathrm{kpc}$ is closer to the plane 


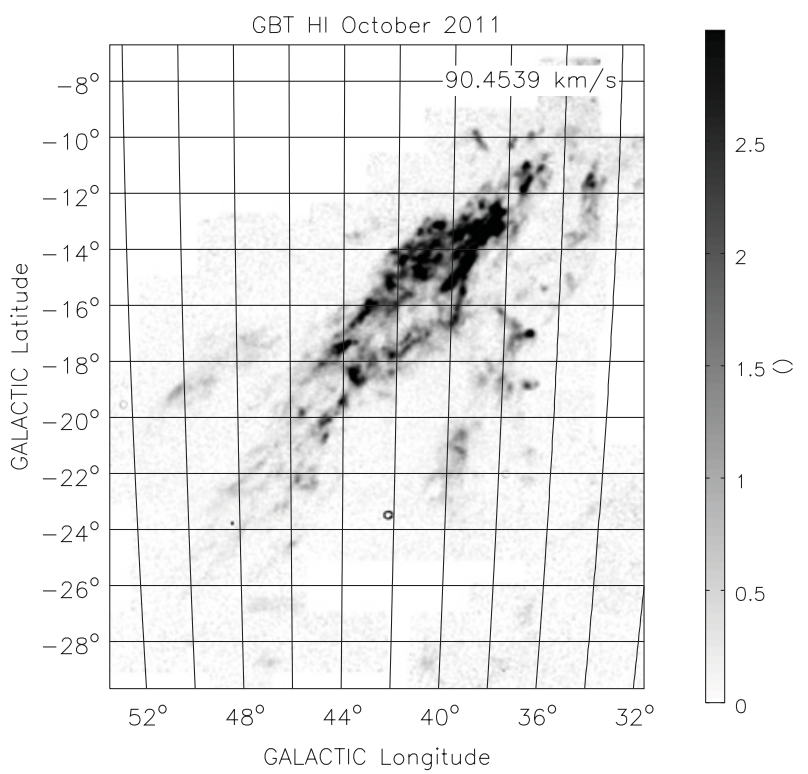

\section{Properties of the Smith Cloud}

distance $=12.4 \pm 1.3 \mathrm{kpc}$

Rgal $=7.6 \pm 1.0 \mathrm{kpc}$

$\mathrm{z}=-2.2 \mathrm{kpc}$

size $\approx 3 \times 1 \mathrm{kpc}$

$\mathrm{M}_{\mathrm{HI}}>10^{6} \mathrm{M}$ 。

$\mathrm{M}_{\mathrm{H}+}>10^{6} \mathrm{M}$ 。

$\mathrm{V}_{\Theta}=264 \pm 21 \mathrm{~km} \mathrm{~s}^{-1}$

$\mathrm{V}_{\mathrm{R}}=113 \pm 21 \mathrm{~km} \mathrm{~s}^{-1}$

$\mathrm{V}_{\mathrm{z}}=70 \pm 25 \mathrm{~km} \mathrm{~s}^{-1}$

Figure 2. HI map of the Smith Cloud at $\mathrm{V}_{\mathrm{LSR}}=90 \mathrm{~km} \mathrm{~s}^{-1}$ from new GBT data. It is moving to the upper right, interacting with the Milky Way halo, and being stripped of its outer parts.

than the top of some superbubbles [14]. Its morphology and detailed kinematics show that it is currently being disrupted and parts of it decellerated to the velocity of the halo gas it is encountering [11]. Optical emission line measurements indicate that it contains at least as much ionized gas as neutral gas, and it has a metallicity below solar [5]. It has exactly the properties needed to fuel future star formation in the Milky Way and account for the chemical evolution of the disk [13]. It is not known to have any stars.

Nichols \& Bland-Hawthorn [12] have modeled the cloud as the baryonic component of $\mathrm{a} \sim 10^{8} \mathrm{M}_{\odot}$ dark matter subhalo, which had lost at least half of its dark and baryonic mass to the Milky Way in an earlier passage through the disk. They estimate that the progenitor had an initial mass that was an order-of-magnitude higher than we observe today.

\subsection{The Smith Stream?}

Is the Smith Cloud a solitary object or might it be part of a larger structure? To examine this we have recently observed a large region of the sky "ahead" of the Smith Cloud in the $21 \mathrm{~cm}$ line with the GBT. Figure 3 shows the HI map that results if at each pixel we display the HI intensity at the velocity which is the projection of $V_{\theta}, V_{R}, V_{z}=+220,+129,+9 \mathrm{~km} \mathrm{~s}^{-1}$, velocity components not too different from that derived for the Smith Cloud. The tip of the Smith Cloud shows up clearly in the lower left of the Figure, and in most pixels there is no $\mathrm{HI}$ emission at the projected velocity. However, the projection reveals a chain of clouds stretching along the trajectory of the Smith Cloud through the Galactic plane. It is possible that these are segments of an extended stream of gas, all following a similar trajectory, of which the Smith Cloud is only the brightest part, and as yet the least disrupted. If so, the "Smith Stream" would have an extent of more than $6 \mathrm{kpc}$.

\section{FUTURE WORK}

The model by Nichols \& Bland-Hawthorn offers one possible explanation for the properties of the Smith Cloud; other work has suggested that HVCs without dark matter halos are disrupted 


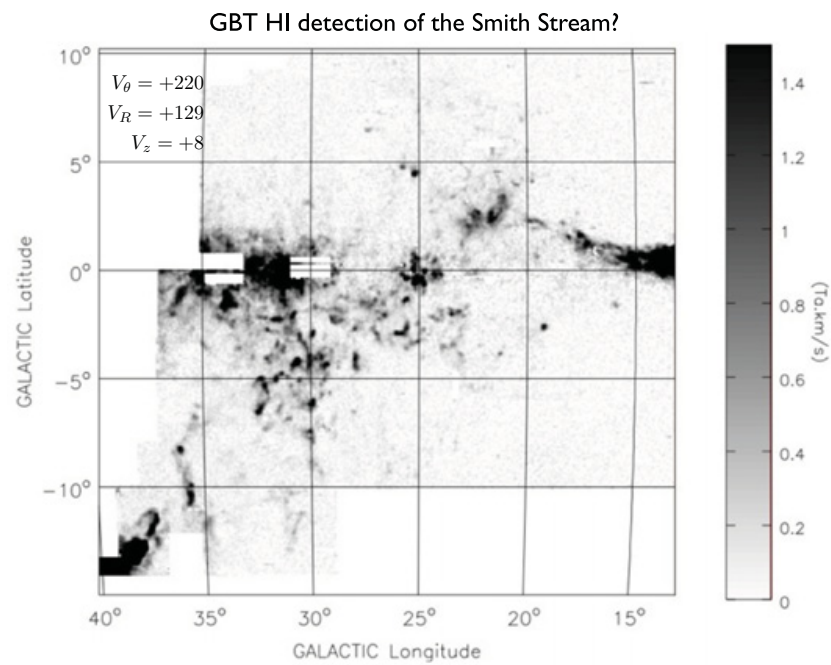

Figure 3. New GBT HI observations of the area ahead of the path of the Smith Cloud (visible in the lower left). This "channel map" shows HI at the velocity calculated from the projection of the velocity components given in the upper left. There is a chain of HI clouds exactly along the predicted path and with kinematics simliar to that of the Smith Cloud, possibly components of the Cloud that are already passing through the Milky Way disk.

significantly before they can reach the disk [6]. This is clearly an area that needs more investigation, and more detailed observations of the Smith Cloud may be able to provide information on the disruption process. The metallicity of the Cloud also needs direct measurement, as current estimates have a large uncertainty [5]. Work is likewise needed on the evolution of superbubbles and the origin of the disk-halo clouds. The Smith Cloud is now traversing the area populated by the diskhalo clouds and it would not be surprising to see signs of its interaction with a lumpy, cloudy, Galactic halo.

\section{References}

[1] Boothroyd, A.I. et al. A\&A (in press: arXiv1110.1765)

[2] Ford, H.A. et al. 2008, ApJ, 688, 290

[3] Ford, H.A., Lockman, F.J., \& McClure-Griffiths, N.M. 2010, ApJ, 722, 367

[4] Grossi, M. et al. 2008, A\&A, 487, 161

[5] Hill, A.S., Haffner, L.M. \& Reynolds, R.J. 2009, ApJ, 703, 1832

[6] Heitsch, F. \& Putman, M.E. 2009, ApJ, 698, 1923

[7] Houck, J.C. \& Bregman, J.N. 1990, ApJ, 352, 506

[8] Howk, J.C., \& Savage, B.D. 2000, AJ, 119, 644

[9] Howk, J.C., \& Savage, B.D. 1999, AJ, 117, 2077

[10] Lockman, F.J. 2002, ApJ, 580, L47

[11] Lockman, F.J., Benjamin, R.A., Heroux, A.J., \& Langston, G.I. 2008, ApJ, 679, L21

[12] Nichols, M., \& Bland-Hawthorn, J. 2009, ApJ., 707, 1642

[13] Pagel, B.E.J. 1994, in The Formation and Evolution of Galaxies, ed. C., Munez-Tunon \& F. Sanchez (Cambridge: CUP), 149

[14] Pidopryhora, Y., Lockman, F.J., \& Shields, J.C. 2007, ApJ, 656, 928

[15] Sancisi, R., Fraternali, F., Oosterloo, T., \& van der Hulst, T. 2008, A\&AR, 15, 189

[16] Shull, J.M. et al. 2011, ApJ, 739, 105 
Assembling the Puzzle of the Milky Way

[17] Smith, G.P. 1963, Bull. Astr. Inst. Netherlands, 17, 203

[18] Stanimirovic, S., et al. 2006, ApJ, 653, 1210

[19] Stil, J.M. et al. 2006, ApJ, 637, 366

[20] Thilker, D. et al. 2004, ApJ, 601, 39

[21] Wakker, B. P. 2001, arXiv:astro-ph/0109210

[22] Wakker, B.P. \& van Woerden, H 1997, ARAA, 35, 217 